\title{
Understanding the mechanisms of root-reinforcement in soils: soil shear tests using X-ray computed tomography and digital volume correlation
}

\author{
Daniel Bull ${ }^{1, *}$, Ian Sinclair ${ }^{1}$, Fabrice Pierron ${ }^{1}$, Tiina Roose $^{1}$, and Joel Smethurst ${ }^{1}$ \\ ${ }^{1}$ Faculty of Physical Sciences and Engineering, University of Southampton, UK
}

\begin{abstract}
Soil containing plant roots may be expected to exhibit a greater shearing resistance compared with the same 'unreinforced' soil, providing enhanced stability and effective erosion control, particularly for earth slopes. To be able to rely on the improved shearing resistance and stiffness of root-reinforced soils, it is important to understand and quantify the effectiveness of root reinforcement. This requires sophisticated multiscale models, building understanding at different length scales, from individual soil-root interaction through to full soil-profile or slope scale. One of the challenges with multiscale models is ensuring that they are representative of real behaviour, and this requires calibration to detailed high-quality experiments. The focus of the work presented was to capture and quantify root-reinforcement behaviour and associated soil and root deformation mechanisms during direct shear at the macroscopic to millimetre length scales. A novel shear box was developed to operate within a large-scale X-ray computed tomography (CT) scanner. Tests were interrupted to be scanned at a series of shear displacements from $0-20 \mathrm{~mm}$ to capture the chronology of behaviour in three-dimensions. Digital volume correlation (DVC) was applied to the CT dataset to obtain full-field 3D displacement and strain component information. The study demonstrates feasibility of the technique and presents preliminary DVC results.
\end{abstract}

\section{Introduction}

The use of vegetation has been demonstrated as an effective approach for improving slope stability to prevent shallow landslides and erosion. The planting of trees, shrubs and grasses on slopes provides a potential increase in soil shear strength due to (1) a reduction in groundwater and pore water pressures as plants transpire water [1], and (2) mechanical root reinforcement of the soil. There are two mechanical effects of roots: (1) small diameter flexible roots that act in tension through soilroot friction to increase the soil-fibre composite strength, and (2) large diameter roots that intersect the shear plane act as individual anchors that can slip through the soil matrix without breaking, mobilizing a small fraction of their tensile strength $[2,3]$.

The magnitude of the root strengthening effect is influenced by many complex factors. These include variabilities in plant roots (geometry, orientation, mechanical properties and root-soil interface) and soil characteristics (compaction, water content, etc). These competing parameters make it challenging to fully understand the behaviour of root-soil interaction and how roots promote the strengthening of soil [2-6].

To quantify the mechanical effects of roots on soil shear strength, there have been several laboratory studies, e.g. direct shear, centrifugal and root tensile strength tests [2-6]. In addition, many models for soilroot interactions have also been developed $[7,8]$.

The direct shear test is a commonly used laboratory method to study the shear strength of soil and root reinforcement. To gather more information from mechanical experiments such as the direct shear test, there have been several studies implementing digital image correlation (DIC) to obtain full-field displacement and shear information during loading. This is achieved using a box with transparent windows through which cameras recorded images of the soil during loading; DIC is applied to the images to obtain displacement and strain fields $[9,10]$. A limitation of this technique is that it can only make observations of the surface plane which could be influenced by the conditions on the boundary. It is also challenging to capture localised features such as soil-root interaction which may not be directly present on the observed surface.

To fully capture the three-dimensional behaviour of the soil and soil-root interaction, it is important to capture information within the bulk of the soil. Developments in advanced X-ray computed tomography (CT) have been shown to work well for capturing 3D information within soils (including roots) and have seen a considerable rise in use in soil science within the past decade [11]. Extending the CT technique further, interrupted in situ experiments on soils have been carried in previous studies to understand the mechanics of soil

\footnotetext{
* Corresponding author: daniel.bull@soton.ac.uk
} 
and soil-root behaviour. These studies have applied digital volume correlation (DVC), a technique similar to DIC to obtain 3D full-field displacement and strain information [12-14] but have so far studies have been limited to high resolution $\mathrm{CT}(<1 \mu \mathrm{m}$ voxel resolution) of small samples [14] or on sand with densely packed fine particles which are easier to track with DVC [12, 13].

The focus of the research presented here is to combine direct shear box experiments with in situ CT to understand and quantify the $3 \mathrm{D}$ soil mechanics and soilroot interactions when subjected to a direct shear load. The study explores the feasibility of this experiment at a larger scale (field of view: $80 \times 80 \times 80 \mathrm{~mm}$ ) to that which has been generally carried out before (both at Southampton and elsewhere) and on soil containing sand, silt and clay with particles up to $2 \mathrm{~mm}$ (representing a suitable growth medium for the plants). Results from these experiments will be used to develop and improve modelling strategies including ongoing work with Meijer et al. [15].

\section{Methodology}

\subsection{Test specimens}

Test specimens comprising of Willow (Salix viminalis, variety Tora) and an unrooted (control) specimen were studied. Test specimens were prepared within a $110 \mathrm{~mm}$ diameter, $\sim 500 \mathrm{~mm}$ long cylindrical tube. The tube comprised of two $250 \mathrm{~mm}$ length sections, forming a plane at the middle of the tube on which controlled shear could later take place. A $2 \mathrm{~mm}$ clearance gap was left between the two tubes, which were supported in position using a purpose-made bracket. The base of the tube was sealed with a permeable membrane.

Both Willow and unrooted specimens were prepared in Bullionfield soil (mineral portions consisting of $71 \%$ sand, $19 \%$ silt and $10 \%$ clay, James Hutton Institute, Dundee, UK) with a pH of 6.2 [3]. The soil was oven dried at $60^{\circ} \mathrm{C}$ for 48 hours and sieved to $<2 \mathrm{~mm}$ particle size. De-aired water was added to achieve a water content of $0.18 \mathrm{gg}^{-1}$. The soil was compacted in 10 equal layers to achieve a target bulk dry density of $1.4 \mathrm{Mg} / \mathrm{m}^{3}$.

The Willow plants were grown for 60 days under controlled lighting and at a controlled temperature of $21^{\circ} \mathrm{C}$ and relative humidity of $41 \%$. Lighting was provided by a Maxibright T5 unit, equipped with eight blue T5 fluorescent tubes delivering 4450 lumens of light per tube, operating for 16 hours per day. The height of the lights was adjusted to maintain a $\sim 150 \mathrm{~mm}$ distance between the lights and the tallest part of the plants.

Immediately prior to carrying out the direct shear tests, specimens were fully saturated in water before being placed on a saturated bed of sand within a large container. Water was drained from the bottom of the container to leave the water table in the sand $0.5 \mathrm{~m}$ below the shear plane in the specimen tubes, creating a water suction of $5 \mathrm{kPa}$ at the shear plane.

\subsection{Test rig}

To facilitate in situ testing within a large-scale X-ray computed tomography (CT) scanner, a bespoke directshear test rig was manufactured (see figure 1). The rig was designed to enable direct control and monitoring of the load, displacement and tensiometer sensors from outside the CT scanner.

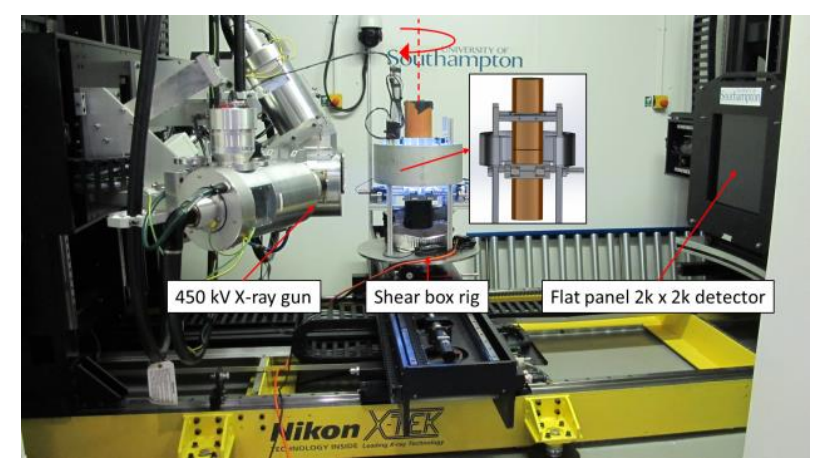

Fig. 1. Experimental setup of the direct shear box test within a large X-ray CT scanner (Hutch) at the University of Southampton.

The test fixture consisted of an aluminium support ring to fully constrain the upper half of the specimen tube whilst providing a consistent X-ray transmission throughout the $360^{\circ}$ rotation. The lower half of the sample tube was mounted to a bracket attached to two guide rails to constrain all degrees of freedom except for the lateral shear displacement.

Once the sample tube was mounted in the rig, the bracket which temporarily supported both halves of the tube was removed. A razor blade set to $2 \mathrm{~mm}$ depth was run around the parameter of the tube at the shear plane to remove the influence of roots in contact with the sidewall of the tube. To minimise the loss of water during the experiment, both the top and bottom of the tubes were covered and taped with aluminium foil to provide an airtight seal, and the stem of the plant above the surface was cut off to remove transpiration effects.

\subsection{X-ray computed tomography: in situ direct shear experiments}

$\mathrm{X}$-ray CT scans were undertaken at the Mu-VIS facilities at the University of Southampton, UK using a large Nikon Metrology Hutch CT scanner.

To provide enough $\mathrm{X}$-ray transmission through the rig and specimens, a $450 \mathrm{kV}$ X-ray gun was used. The scan settings are shown in table 1 . A short scan time was chosen to minimise the effects of soil movement during the scan, and to enable all scans to be undertaken within a 12-hour session.

CT scans consisted of four noise study scans and seven shear test scans. Noise study scans were used to provide a noise sensitivity study for DVC. These consisted of two stationary scans, one rigid body motion scan with the specimen displaced $2 \mathrm{~mm}$ upward and one change in magnification scan, with the specimen displaced $2 \mathrm{~mm}$ away from the X-ray gun. In the first two cases (stationary and rigid body motion), the 
expected strains should be zero. In the last case, the magnification will provide an artificial uniform increase in strain.

Table 1. X-ray CT scan parameters.

\begin{tabular}{|c|c|}
\hline CT scan parameter & Value \\
\hline Voltage & $300 \mathrm{kV}$ \\
\hline Power & $90 \mathrm{~W}$ \\
\hline Voxel resolution & $46 \mu \mathrm{m}$ \\
\hline Number of projections & 3142 \\
\hline Frames per projection & 4 \\
\hline Exposure time & $134 \mathrm{~ms}$ \\
\hline Scan time & 30 minutes \\
\hline Filtering & $\begin{array}{l}8 \mathrm{~mm} \text { aluminium (total } \\
\text { transmission through wall } \\
\text { thickness from cylindrical } \\
\text { support on test rig) }\end{array}$ \\
\hline
\end{tabular}

The seven shear test scans consisted of a scan with the tube in its initial (unloaded) position, followed by six scans performed after an incremental shear displacement of $\sim 3.33 \mathrm{~mm}$ had been applied, to provide a total shear displacement of $20 \mathrm{~mm}$. After application of each shear displacement, specimens were held at this displacement for $\sim 30$ minutes to allow for stress-relaxation, i.e. a small drop in recorded load. This was done to minimise any movement of the specimen during the CT scan.

\subsection{Digital volume correlation (DVC)}

Volumetric CT data was processed using DaVis LaVision software to obtain DVC data. Matching between sub-volumes was done using a coarse fast Fourier transform correlation step to create an initial predictor of the displacement field followed by a direct correlation step.

The DVC process involves choosing an adequate subset size to achieve a balance of noise, reliable correlation, strain measurement accuracy and spatial resolution. It is therefore important to first perform a noise sensitivity study to evaluate the strain measurement resolution [16]. The strain measurement resolution will determine the minimum significant strain value that can be extracted from the deformed images which can be associated with material behaviour rather than noise artefacts.

For the noise study, five subsets with $75 \%$ overlap were studied with varying isotropic sub-volume sizes with equal length, width and depths of: 192, 128, 96, 64, 48 , and 32 pixels. Figure 2 shows a plot containing the standard deviation of Green-Lagrangian strain components $\varepsilon_{\mathrm{xx}}, \varepsilon_{\mathrm{yy}}$ and $\varepsilon_{\mathrm{zz}}$ for a stationary, rigid body displacement and magnification noise study scan. Results show a knee point at a subset size of $\sim 64 \mathrm{px}$ corresponding to a standard deviation of strain between 1000-1500 microstrain.

For the incrementally loaded specimens, trials were conducted to establish how well the deformed volumes correlated. Due to the large deformations in this dataset, it was necessary to apply a sum of differential approach instead of the reference to first method. In the latter technique, each displacement step from 0 to $20 \mathrm{~mm}$ was referenced against the initial unloaded volume. The first shear displacement step of $3.3 \mathrm{~mm}$ showed strong correlation, however there was a breakdown in correlation in subsequent displacement steps $\geq 6.6 \mathrm{~mm}$ leading to inaccuracies when compared to an LVDT sensor measuring the displacement of the specimen in the test rig. In contrast, the sum of differential approach performs volumetric correlation between each loading step rather than correlating against the first reference image. The incremental correlations between steps are then summed together to obtain the deformation fields. Whilst the sum of differential approach showed strong correlation and displacement measurement accuracy between 0 to $20 \mathrm{~mm}$ shear displacements, it should be noted that the noise (as illustrated in figure 2) will be summed together at each load step. Therefore, it is expected that the strain resolution for the final sheardisplacement step will be $\sim 7$ times larger than shown in the noise study experiment.
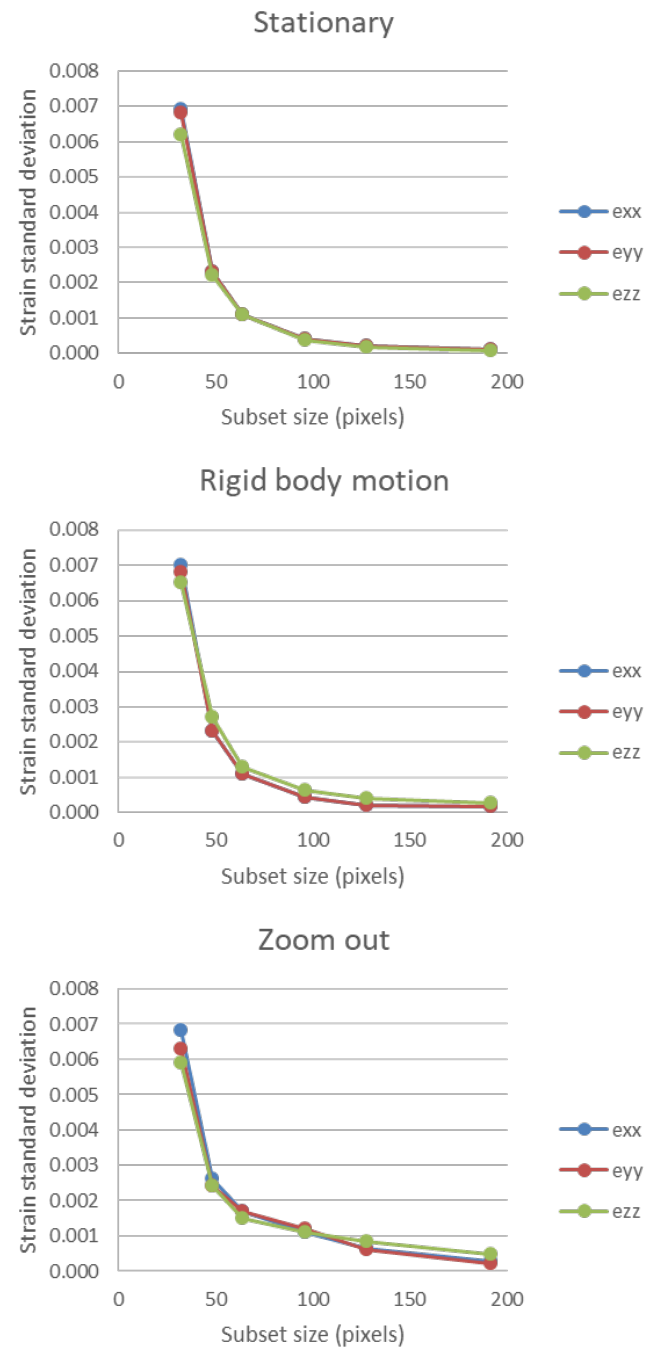

Fig. 2. DVC noise study on soil scan data comparing the effects of varying subset size.

After conducting the noise study test and preliminary DVC trials, a sub-volume size of 32 pixels, $75 \%$ overlap and sum of differential approach was chosen to process the shear load scan data. Despite the high noise 
associated with the 32 pixel subset, there was a strong signal-to-noise on the shear-loaded scans with strain signals within the shear band zone exceeding 10 times the noise. In this case, the smaller 32 pixel subset size improved the spatial resolution of the data and enabled localised strain features to be captured. A smaller 24 pixel subset size was trialled, however there was poor correlation. This was due to computer hardware limitations which restricted the search radius size in the direct correlation step.

\section{Preliminary $\mathrm{CT}$ and digital volume correlation results}

The short 30-minute CT scans at a voxel resolution of 46 $\mu \mathrm{m}$, offered good resolution and contrast to capture the soil particles, pore space and tap roots. Figure 3 shows a cross-sectional CT slice (top view) at the shear plane. Three tap roots are visible (circled regions) and are observed growing vertically into the tube (into the page).

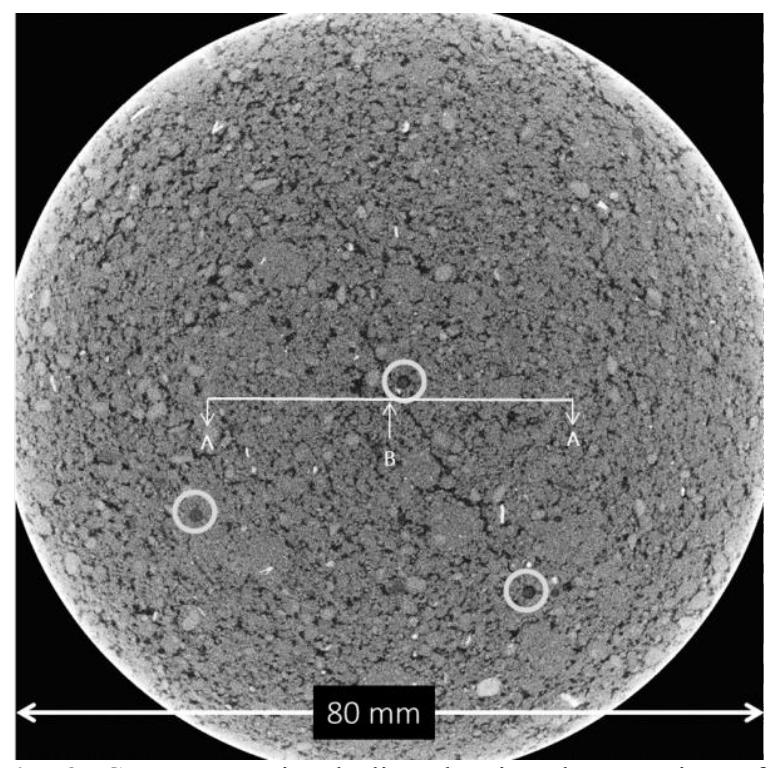

Fig. 3. CT cross-sectional slice showing the top view of a Willow planted specimen at the shear plane. Circled are three tap roots with a diameter $\sim 1.5 \mathrm{~mm}$ growing into the page.

DVC was used to process shear-displacement volumes for both willow and unrooted specimens. The processed data produced volumetric full-field displacements and Green-Lagrangian strain component data.

Figure 4 shows a displacement profile plot taken at position $\mathrm{B}$ indicated in figure 3 (near the vicinity of the root), with the column depth axis extending into the page. The line plot shows the shear displacement position vs. tube depth above and below the shear plane. Above the shear plane, the minimum displacement approaches $0 \mathrm{~mm}$ and is representative of the fully constrained half of the tube, whereas below the shear plane, the maximum displacement approaches $20 \mathrm{~mm}$, i.e. the displaced half of the tube.

Comparing the displacement profile of both willow and the unrooted specimens, there is a difference in the shape of the profile between the two systems. The willow exhibits a near linear displacement transition through the shear band, whereas the unrooted specimen exhibits an exponential bias towards the shear plane.

It is interesting to point out that the thickness of the shear band in both specimens is approximately $30 \mathrm{~mm}$. Comparing the willow displacement profile to the $\mathrm{CT}$ image of the deformed root shown in figure 4 , it is observed that the geometry of root displacement follows the pattern of soil displacement.

Whilst the size of the shear zones for both willow and unrooted specimens are near identical, an interesting observation is made when the volumetric strains (sum of $\varepsilon_{\mathrm{xx}}, \varepsilon_{\mathrm{yy}}$ and $\varepsilon_{\mathrm{zz}}$ ) are compared. Figure 5 shows a full-field profile of the volumetric strains taken at section profile A-A indicated in figure 3 . In both specimens there is a local increase in volumetric strain at the shear band which is associated with soil dilation, however there are differences in the magnitude and distribution of volumetric strains. The unrooted system shows a near consistent region of high $(>2)$ volumetric strain across the shear band, whereas a heterogeneous distribution is observed in the willow sample.

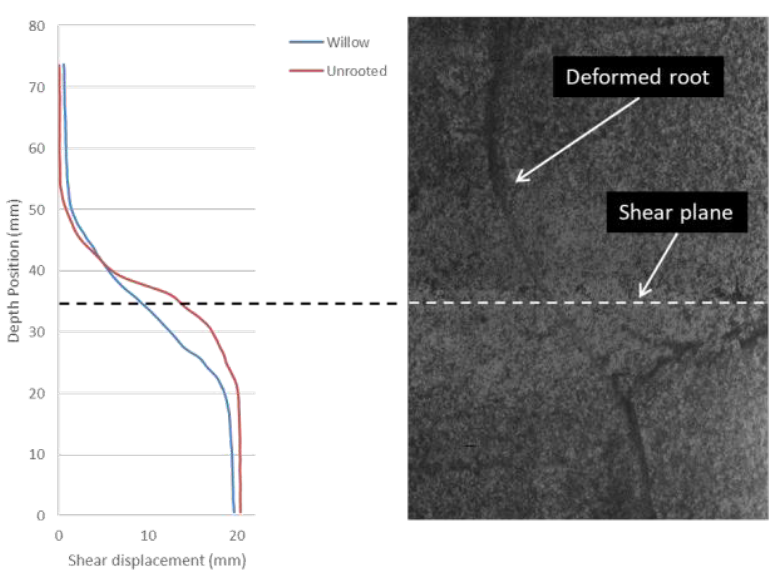

Fig. 4. (Left) plot of local shear displacement vs. depth above and below the shear plane after application of $20 \mathrm{~mm}$ shear displacement. (Right) cross-sectional slice showing a deformed vertical root following the shape of the shear band.
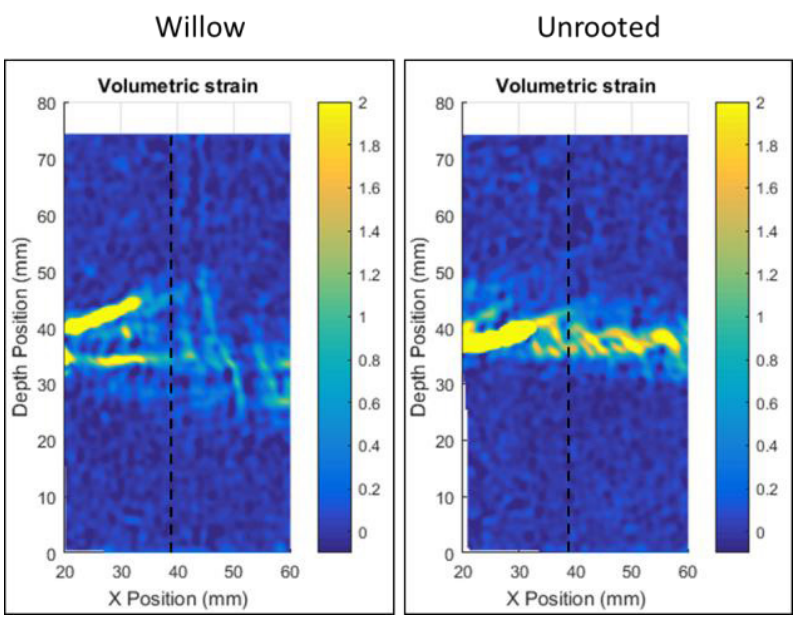

Fig.5. Full-field volumetric strains after application of $20 \mathrm{~mm}$ shear displacement. Image taken at section profile A-A (indicated on figure 3). 
To illustrate the difference in volumetric strain magnitude between the two systems, a line profile of the dotted lines shown in figure 5 is presented in figure 6 . Within this region, there is a twofold increase in volumetric strain on the unrooted sample compared to the willow specimen. In other areas, this difference was observed to exceed 5 times. One possible explanation for the difference in peak volumetric strain between the two specimen is associated with the constraining effects of the roots, which once mobilised locally suppress the magnitude of dilation. At the extremities of the willow specimen in figure 5 (left side at shear band), the peak magnitude of dilation matches that of the unrooted sample suggesting the roots have a local area of influence on the surrounding soil.

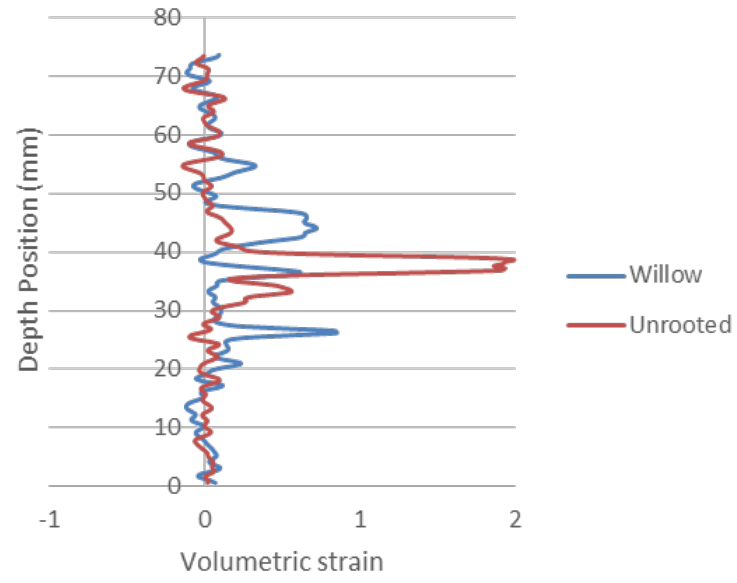

Fig.6. Line profile of volumetric strains between willow and unrooted specimens, taken from the dotted line in figure 5.

\section{Concluding remarks and future work}

Preliminary results have demonstrated the feasibility of conducting DVC experiments on direct shear tests of root-reinforced soil at this scale $(80 \times 80 \times 80 \mathrm{~mm}$ field of view). An externally controlled, fully instrumented in situ test rig was demonstrated to work successfully in this application.

The large deformations during the shear box test $(0$ $20 \mathrm{~mm}$ ) required a sum of differential approach to provide accurate results from DVC data. For this setup (soil type, voxel resolution, and sub-volume size), an intermediate shear-displacement step size of $\leq 3.3 \mathrm{~mm}$ was necessary to ensure strong correlation between volumes.

Initial results from DVC data show that full-field displacements and strains can be captured. It was observed that the roots follow the overall shear displacement path within a $\sim 30 \mathrm{~mm}$ thick shear band zone. Comparisons between unrooted and willow specimens show an identical shear zone size, although there are differences to the shear displacement profile across this zone. As expected [17], an increase in volumetric strain associated with dilation was observed at the shear band. However, comparisons between unrooted and willow specimens show differences in the magnitude and distribution of volumetric strains.
Ongoing research will assess DVC data in more detail to further understand the local effects of soil-root interaction. Results will also be used to inform the correct mechanisms and provide quantification to validate ongoing model development [15].

This research was funded by EPSRC grant EP/M020355/1, part of a collaboration between the University of Southampton, University of Dundee, University of Aberdeen, Durham University and the James Hutton Institute. The James Hutton Institute receives funding from the Scottish Government (Rural \& Environmental Services \& Analytical Services Division).

\section{References}

1. J. Smethurst D. Clarke, W. Powrie, Geotechnique, (62), 429-446 (2006)

2. E. Comino and A. Druetta, American J. of Environmental Sciences, 5 (4), 475-486 (2009)

3. T. Liang, A.G. Bengough, J. Knappett, D. Muir Wood, K.W. Loades, P.D. Hallett, D. Boldrin, A.K. Leung, G.J. Meijer, Ecol Eng 109, 207 (2017)

4. T. H. Wu and A. Watson, Canadian Geotechnical Journal, 35(4), 579-590 (1998)

5. D. Hengchaovanich and N. S. Nilaweera, Proceedings of First Int. Vetiver Conf, Bangkok, Thailand, 153-158 (1996)

6. G. Veylon, M. Ghestem, A. Stokes and A. Bernard, Canadian Geotechnical Journal, 52(11), 1839-1849 (2015)

7. L. J. Waldron, Soil Sci Soc. Am. J., 41, 842-849 (1977)

8. G.J. Meijer, D. Muir Wood, J.A. Knappett, A.G. Bengough, T. Liang, Int J Numer Anal Met (2018), accepted for publication

9. M. P. McGuire, Proceedings of the $122^{\text {nd }}$ ASEE annual conference \& exposition, Seattle, USA (2015)

10. S. A. Hall, D. M. Wood and E. Ibraim, Granular Matter, 12, 1-14 (2010)

11. I. A. Taina, R. J. Heck and T. R. Elliot, Canadian J. of Soil Science, 88(1), 1-19 (2008)

12. S. A. Hall, M. Bornert, J. Desrues, Y. Pannier, N. Lenoir, G. Viggiani and P. Desuelle, Geotechnique, 60(5), 315-322 (2010)

13. D. Takano, N. Lenoir, J. Otani and S. A. Hall, Soils and Foundations, 55(4), 906-915 (2015)

14. S. D. Keyes, L. Cooper, S. Duncan, N. Koebernick, D. M. McKay Fletcher, C. P. Scotson, A. van Veelen, I. Sinclair and T. Roose, J. of the Royal Society Interface, 136 (14), (2017)

15. G. Meijer, D. Wood, J. Knappett, G. Bengough and T. Liang. Proceedings of the 7th International Symposium on Deformation Characteristics of Geomaterials, Glasgow, UK, (2019)

16. F. Gillard, R. Boardman, M. Mavrogordato, D. Hollis, I. Sinclair, F. Pierron, M. Browne, J. 
Mechanical Behaviour of Biomedical Materials 29,

480-499 (2014)

17. M. D. Bolton, Geotechnique 39, 65-78 (1986) 